\title{
SOME SPECIAL LIMITS OF SCHOTTKY GROUPS
}

\author{
DANIEL M. GALLO
}

(Communicated by Clifford J. Earle, Jr.)

\begin{abstract}
We show that every extended Schottky group $G$ which uniformizes a compact Riemann surface $S$ is a geometric limit of Schottky groups $G_{n}$ which also uniformize $S$. That is, every element $g \in G$ is the limit of elements $g_{n} \in G_{n}$.
\end{abstract}

Let $\Gamma$ be a Fuchsian group covering a compact Riemann surface $S$, and let $(f, \chi)$ be a projective structure on $S$. Suppose that $f$ is a covering map and that $\chi(\Gamma)$ is an extended Schottky group. (Definitions will be given in the text. For the moment, we note that our definition of extended Schottky group does not include Schottky groups.) In this paper, we will show that there exists a sequence $\left(f_{n}, \chi_{n}\right)$ of projective structures on $S$ with the following properties: (i) $\chi_{n}(\Gamma)$ is a Schottky group, and (ii) $\left(f_{n}, \chi_{n}\right)$ converges to $(f, \chi)$. The precise statement, Theorem 1, and proof of this result are given in $\S 3$. In $\S 4$ we prove an analogue of Theorem 1 for compact surfaces of genus 1 .

We note that the homomorphisms $\chi_{n}$ converge algebraically to $\chi$. In our proofs, we exhibit cyclic subgroups of $\chi_{n}(\Gamma)$ which have a rank 2 parabolic subgroup of $\chi(\Gamma)$ as a geometric limit (Lemma 1 and Theorem 2). This reproduces, within the context of algebraic convergence of projective structures on a fixed Riemann surface, the basic example of a geometric limit given by Jorgensen and Thurston (see [9] for details).

\section{Preliminaries}

Throughout, $\Gamma$ will denote a torsion-free Fuchsian group acting on the upper half plane $U$ and covering a compact surface $U / \Gamma$. We denote the space of holomorphic quadratic differentials (defined on $U$ ) for $\Gamma$ by $B_{2}(\Gamma)$ and equip $B_{2}(\Gamma)$ with the norm $\|\varphi\|=\sup _{z \in U}\left\{|\varphi(z)|(2 \operatorname{Im} z)^{2}\right\}$.

For every $\varphi \in B_{2}(\Gamma)$ there exists a meromorphic local homeomorphism $f_{\varphi}=$ $f$, where $f: U \rightarrow \widehat{\mathbf{C}}$ and $S(f)=\varphi$; here $S(f)$ is the Schwarzian derivative of $f$ given by

$$
S(f)=\left(f^{\prime \prime} / f^{\prime}\right)^{\prime}-\frac{1}{2}\left(f^{\prime \prime} / f^{\prime}\right)^{2} .
$$

Received by the editors March 25, 1991 and, in revised form, November 14, 1991; the contents of this paper were presented to the 860th Meeting of the American Mathematical Society at the University of Massachusetts, Amherst, MA, October, 1990.

1991 Mathematics Subject Classification. Primary 30F10; Secondary 57M99. 
The function $f$ determines a homomorphism $\chi_{\varphi}: \Gamma \rightarrow \operatorname{PSL}(2, \mathbf{C})$ with the defining property $f \circ \gamma(z)=\chi_{\varphi}(\gamma) \circ f(z)$ for all $\gamma \in \Gamma, z \in U$.

The pair $\left(f, \chi_{\varphi}\right)$ is called a projective structure on $U / \Gamma$. In general, we will require that $f$ be normalized by the conditions $f(i)=0, f^{\prime}(i)=1, f^{\prime \prime}(i)=0$. With this normalization, there is a one-to-one correspondence between projective structures on $U / \Gamma$ and elements of $B_{2}(\Gamma)$ (see $[5,7]$ for details). We will restrict our attention in this paper to the subset $C_{2}(\Gamma) \subset B_{2}(\Gamma)$ defined by

$$
C_{2}(\Gamma)=\left\{\varphi \in B_{2}(\Gamma): f \text { is a covering map }\right\} .
$$

It was shown by Kra-Maskit [7] that $C_{2}(\Gamma)$ is a closed and bounded subset of $B_{2}(\Gamma)$. We note that, for $\varphi \in C_{2}(\Gamma), \chi_{\varphi}(\Gamma)$ is a Kleinian group with $f(U)$ an invariant component (see [5]).

\section{UNIFORMIZATIONS OF SCHOTTKY TYPE}

By a quadrilateral $Q$ in the extended complex plane $\widehat{\mathbf{C}}$, we will mean a Jordan curve $Q \subset \widehat{\mathbf{C}}$ on which four distinct vertices have been chosen so as to yield opposite pairs of sides $a, a^{\prime}$ and $b, b^{\prime}$. Let $\alpha, \beta \in \operatorname{PSL}(2, \mathbf{C})$ be parabolic transformations with a common fixed point contained in one of the complementary components of $Q$ and suppose that $\alpha$ and $\beta$ identify opposite sides of $Q$ so that $J=\langle\alpha, \beta\rangle$ is a rank 2 parabolic group. We will say that $J$ is a parabolic group associated to $Q$.

Let $Q_{1}, \ldots, Q_{p}, C_{p+1}, \ldots, C_{p+q}, C_{p+1}^{\prime}, \ldots, C_{p+q}^{\prime}$ be a collection of mutually disjoint Jordan curves in $\widehat{\mathbf{C}}$ that bound a connected set $F$. Suppose $Q_{i}, i=1, \ldots, p$, are quadrilaterals with opposite pairs of sides $a_{i}, a_{i}^{\prime}$ and $b_{i}, b_{i}^{\prime}$ and associated parabolic groups $J_{i}=\left\langle\alpha_{i}, \beta_{i}\right\rangle$, which have their fixed points in the interior of $Q_{i}$ (that component not intersecting $F$ ). Let $\alpha_{i}\left(b_{i}\right)=$ $b_{i}^{\prime}, \beta_{i}\left(a_{i}\right)=a_{i}^{\prime}$.

Furthermore, suppose $\gamma_{j}, j=p+1, \ldots, p+q$, are loxodromic transformations which map the interior of $C_{j}$ onto the exterior of $C_{j}^{\prime}$. Denote by $G$ the free product $J_{1} \times \cdots \times J_{p} \times\left\langle\gamma_{p+1}\right\rangle \times \cdots \times\left\langle\gamma_{p+q}\right\rangle$. We will say that $G$ is a group of Schottky type if $p+q>1$. A group of Schottky type is an extended Schottky group if $p>0$; it is a Schottky group if $p=0$. (We note that this terminology is not standard.) It is well known that $G$ is a Kleinian group with a connected region of discontinuity $\Omega(G)$.

The compact surface $\Omega(G) / G$ has genus $p+q$ and the natural projection $\pi: \Omega(G) \rightarrow \Omega(G) / G$ is an unbranched covering map. The images $\pi\left(a_{i}\right)$ and $\pi\left(b_{i}\right)$ correspond to simple closed curves which are homologically independent; $\pi\left(a_{i}\right)$ goes around the $i$ th hole, and $\pi\left(b_{i}\right)$ goes around the $i$ th handle. $\pi\left(C_{j}\right)$ is a simple closed curve which goes around the $j$ th hole.

\section{StATEMENT AND PROOF OF MAIN RESUlT}

Given a Kleinian group $G$ with an invariant component $\Omega(G)$, let $h$ : $U \rightarrow \Omega(G)$ be a holomorphic universal covering map. The Fuchsian equivalent $\Gamma$ of $G$ is defined by

$$
\Gamma=\{\gamma \in \operatorname{PSL}(2, \mathbf{C}): \exists g \in G \text { with } h \circ \gamma=g \circ h\} .
$$

Define $\chi: \Gamma \rightarrow G$ by $\chi(\gamma)=g$ whenever $h \circ \gamma=g \circ h$. It follows that $U / \Gamma=$ $\Omega(G) / G$, that $(h, \chi)$ is a projective structure on $\Gamma$, and that $S(h) \in C_{2}(\Gamma)$. 
Precomposing $h$ with a suitable Möbius transformation $T$, and conjugating $G$ by $T$, we may assure that $h$ is normalized.

Theorem 1. Let $\varphi \in C_{2}(\Gamma)$ with $\chi_{\varphi}(\Gamma)$ an extended Schottky group. Then there exists a sequence $\varphi_{n} \in C_{2}(\Gamma), \varphi_{n} \rightarrow \varphi$, with $\chi_{\varphi_{n}}(\Gamma)$ a Schottky group.

In order to prove the theorem we will use a preliminary construction and four lemmas. Let $(h, \chi)$ be a projective structure on $U / \Gamma$, where $\Gamma$ is the Fuchsian model of an extended Schottky group $\chi(\Gamma)$. The structure $(h, \chi)$ will remain fixed from now on. As described in $\S 2$, we have associated to $\chi(\Gamma)$ quadrilaterals $Q_{i} \subset \widehat{\mathbf{C}}$ and Jordan curves $C_{j}, C_{j}^{\prime}, i=1, \ldots, p, j=p+$ $1, \ldots, p+q$. (We will avail ourselves of all the notation established in $\S 2$.) Let $z_{0} \in \Omega(\chi(\Gamma)) / \chi(\Gamma)=S$ and choose simple loops $A_{i}, B_{i} \in \pi_{1}\left(S, z_{0}\right)=\pi_{1}(S)$ homologous to $\pi\left(a_{i}\right)$ and $\pi\left(b_{j}\right)$, respectively. Choose $A_{j} \in \pi_{1}(S)$ to be a simple loop homologous to $\pi\left(C_{j}\right)$. It is well known (see, e.g., Hejhal [2]) that we may choose simple loops $B_{j} \in \pi_{1}(S)$ so that $\left\{A_{i}, B_{i} ; A_{j}, B_{j}\right\}$ is a canonical set of generators for $\pi_{1}(S)$. That is, $\prod_{i=1}^{p}\left[A_{i}, B_{i}\right] \prod_{j=p+1}^{p+q}\left[A_{j}, B_{j}\right]=$ id. (Here, $[A, B]$ denotes the commutator $A B A^{-1} B^{-1}$.)

Identifying $\pi_{1}(S)$ with $\Gamma$ so that we have $\chi\left(A_{i}\right)=\alpha_{i}, \chi\left(B_{i}\right)=\beta_{i}$, and $\chi\left(B_{j}\right)=\gamma_{j}$, we also have from uniformization theory (see [8]) $\operatorname{ker} \chi=$ $\left\langle\left\langle A_{i} B_{i} A_{i}^{-1} B_{i}^{-1} ; A_{j}\right\rangle\right\rangle=N$. Here, and elsewhere, $\langle\langle\cdot\rangle\rangle$ denotes the normal subgroup of $\Gamma$ generated by the given arguments.

We now construct a sequence of projective structures on $U / \Gamma$. Observe that, for each $n \geq 0,\left\{A_{i} B_{i}^{n}, B_{i} ; A_{j}, B_{j}\right\}$ is a canonical set of generators for $\Gamma$. Let $N_{n}=\left\langle\left\langle A_{i} B_{i}^{n} ; A_{j}\right\rangle\right\rangle$. It is a classical result (see [2]) that $N_{n}$ determines a projective structure $\left(f_{n}, \chi_{n}\right)$ on $U / \Gamma$, with $f_{n}$ normalized, where $G_{n}=$ $\chi_{n}(\Gamma)=\Gamma / N_{n}$ is a Schottky group. The region of discontinuity of $G_{n}$ is $f_{n}(U)$ and $\chi_{n}\left(B_{i}\right), \chi_{n}\left(B_{j}\right)$ are free generators for $G_{n}$. Moreover, $f_{n}(U) / G_{n}=U / \Gamma$, $\Gamma$ is the Fuchsian model of $G_{n}$, and $\operatorname{ker} \chi_{n}=N_{n}$. We note that the groups $N_{n}$ are distinct. This last assertion is easily verified by passing to $\bar{N}_{n}$, the projection of $N_{n}$ in homology, and noting that the groups $\bar{N}_{n}$ are distinct. It follows that the structures $\left(f_{n}, \chi_{n}\right)$ are distinct (see $\left.[2,8]\right)$.

Now $S\left(f_{n}\right) \in C_{2}(\Gamma)$, and, applying the results of [7], we choose a subsequence $\left(f_{n}, \chi_{n}\right)$ and a projective structure $\left(f, \chi^{*}\right)$ on $\Gamma$ such that $S\left(f_{n}\right) \rightarrow S(f)$. It follows that $f_{n} \rightarrow f$ uniformly on compact subsets of $U$, and $\chi_{n} \rightarrow \chi^{*}$ algebraically. Note that $f$ is normalized and $S(f) \in C_{2}(\Gamma)$. Our objective is to show that $\operatorname{ker} \chi^{*}=\operatorname{ker} \chi \quad($ Lemma 4).

It is a consequence of a result of Jorgensen-Klein [4] (see also, Jorgensen [3] or $\mathrm{Kra}[6])$ that the map $\theta_{n}: \chi^{*}(\Gamma) \rightarrow \chi_{n}(\Gamma)$ which sends $\chi^{*}(\gamma)$ to $\chi_{n}(\gamma)$, $\gamma \in \Gamma$, is a homomorphism for almost all $n$. Thus, $\chi_{n}=\theta_{n} \circ \chi^{*}$, and one has

$$
\operatorname{ker} \chi^{*} \subset \operatorname{ker} \chi_{n} \text {. }
$$

Although we will not need the full strength of the next lemma, we include it for clarity.

Lemma 1. For a fixed $i, i=1, \ldots, p, \chi^{*}\left(A_{i}\right), \chi^{*}\left(B_{i}\right)$ are parabolic and generate a rank 2 parabolic group.

Proof. For simplicity, we drop the subscript $i$. For each $n, \chi_{n}\left(A B^{n}\right)=$ id and $\chi_{n}(A)=\chi_{n}\left(B^{-n}\right)$. Since $\chi_{n}(A) \rightarrow \chi^{*}(A)$, it follows that $\chi_{n}\left(B^{-n}\right) \rightarrow \chi^{*}(A)$. In particular, $\chi_{n}\left(B^{-n}\right)$ converges. 
Since $\chi_{n}(B) \neq$ id for all $n$ and $\chi_{n}(\Gamma)$ is a free group, we have that $\chi_{n}\left(B^{m}\right) \neq$ id for all $m \neq 0$. Thus, applying $(3.1), \chi^{*}\left(B^{m}\right) \neq$ id and $\chi^{*}(B)$ is neither elliptic nor trivial. Similarly, since $\chi_{n}(A)=\chi_{n}\left(B^{-n}\right) \neq \mathrm{id},(3.1)$ yields that $\chi^{*}(A)$ is neither trivial nor elliptic.

Hence, $\chi^{*}(B)$ is either loxodromic or parabolic. Suppose it is loxodromic. Letting $\lambda_{n}, \lambda \in \mathbf{C}$, with $\left|\lambda_{n}\right|,|\lambda|>1$, be the multipliers of $\chi_{n}(B)$ and $\chi^{*}(B)$, respectively, it follows easily that $\left|\lambda_{n}\right| \rightarrow|\lambda|$; but, since $\chi_{n}\left(B^{-n}\right)$ converges, we also have that $\left|\lambda_{n}\right|^{n}$ converges. This contradiction establishes that $\chi^{*}(B)$ is parabolic. Since the fixed points of $\chi_{n}\left(B^{-n}\right)$ coincide with the fixed points of $\chi_{n}(B)$, they must also converge to the fixed point of $\chi^{*}(B)$. Hence, $\lim \chi_{n}\left(B^{-n}\right)=\chi^{*}(A)$ is parabolic and $\chi^{*}(A), \chi^{*}(B)$ have a common fixed point.

To prove that $\chi^{*}(A), \chi^{*}(B)$ generate a rank 2 parabolic group, it suffices to show that $\chi^{*}\left(A^{s}\right) \neq \chi^{*}\left(B^{t}\right)$ for all integers $s, t \neq 0$. Now, for fixed $s, t \neq 0$, $t=-n s$ is satisfied by at most one value of $n$. Hence, for almost all $n$, $\chi_{n}\left(A^{s} B^{-t}\right)=\chi_{n}\left(B^{-n s} B^{-t}\right) \neq$ id. Applying (3.1), we have that $\chi^{*}\left(A^{s} B^{-t}\right) \neq \mathrm{id}$, and the lemma follows.

Given $r$ a fixed integer, $r=1, \ldots, p+q$, the nontrivial words $w\left(A_{r}, B_{r}\right) \in$ $\Gamma$ in the generators $A_{r}, B_{r}$ may be written as

$$
w\left(A_{r}, B_{r}\right)=\prod_{k=1}^{s} A_{r}^{m_{k}} B_{r}^{n_{k}} .
$$

Here, $s \geq 1$ and at least one of the integers $m_{1}, n_{1} \neq 0$. If $s>1$, then $n_{1} \neq 0, m_{s} \neq 0$, and $m_{k}, n_{k} \neq 0$, for $1<k<s$. The length of $w$, denoted by $|w|$, is $s$. Define $w^{A}=\sum_{k=1}^{s} m_{k}$ and $w^{B}=\sum_{k=1}^{s} n_{k}$. For $r=1, \ldots, p$, we will write $[w]=0$, if $w^{A}=w^{B}=0$. For $r=p+1, \ldots, p+q$, we will write $[w]=0$, if $w^{B}=0$.

Lemma 2. Let $i$ be a fixed integer, $i=1, \ldots, p$, and $w\left(A_{i}, B_{i}\right) \in \Gamma$ a nontrivial word with $[w]=0$. Then $w \in\left\langle\left\langle A_{i} B_{i} A_{i}^{-1} B_{i}^{-1}\right\rangle\right\rangle=K_{i}$.

Proof. Dropping the subscript $i$, we write $w=\prod_{k=1}^{s} A^{m_{k}} B^{m_{k}}$ as in (3.2). The proof is by induction on $s$. First, consider the induced map $\theta: \Gamma \rightarrow \Gamma / K$, and note that $\theta(A), \theta(B)$ commute. Since $\Gamma / K$ has a representation as a discrete, torsion-free subgroup of $\operatorname{PSL}(2, \mathrm{C})$ (see [8]), we have that all powers of $\theta(A), \theta(B)$ commute. Hence, $A^{m} B^{n} A^{-m} B^{-n} \in K$ for all integers $m, n$. It follows that the lemma is true for $s=1,2$.

We assume the lemma holds for all $k<s$ with $s>2$. After conjugating by $B^{n_{s}}$ (that is, taking $B^{n_{s}} w B^{-n_{s}}$ ) and reindexing, we assume $n_{s}=0$.

Case 1. $m_{1} \neq 0$. Since $s>2$, we write $w=A^{m_{1}} B^{n_{1}} A^{m_{2}} B^{n_{2}} \cdots A^{m_{s}}$. Thus, $w=B^{n_{1}} w_{1} A^{m_{1}+m_{2}} B^{n_{2}} \cdots A^{m_{s}}$, where $w_{1}=B^{-n_{1}} A^{m_{1}} B^{n_{1}} A^{-m_{1}}, \quad w_{1} \in K$, and $B^{-n_{1}} w B^{n_{1}}=w_{1} w_{2}$, where $w_{2}=A^{m_{1}+m_{2}} B^{n_{2}} \cdots A^{m_{s}} B^{n_{1}}$. Now, $\left|w_{2}\right|=$ $s-1$ and $\left[w_{2}\right]=0$ and, by the inductive hypothesis, $w_{2} \in K$. Hence, $w=$ $B^{n_{1}} w_{1} w_{2} B^{-n_{1}} \in K$.

Case 2. $m_{1}=0$. Here, $w=B^{n_{1}} A^{m_{2}} B^{n_{2}} \cdots A^{m_{s}}$, and letting $w_{1}=B^{-n_{1}} w B^{n_{1}}$ $=A^{m_{2}} B^{n_{2}} \cdots A^{m_{s}} B^{n_{1}}$, we have $\left|w_{1}\right|=s-1,\left[w_{1}\right]=0$. By the inductive hypothesis, $w_{1} \in K$, and it follows that $w \in K$.

Lemma 3. Let $j$ be a fixed integer, $j=p+1, \ldots, p+q$, and $w\left(A_{j}, B_{j}\right) \in \Gamma$ a nontrivial word with $[w]=0$. Then $w \in\left\langle\left\langle A_{j}\right\rangle\right\rangle=K_{j}$. 
Proof. Dropping the subscript $j$, we write $w=\prod_{k=1}^{s} A^{m_{k}} B^{n_{k}}$ and define $\bar{t}=$ $\sum_{k=1}^{t} n_{k}, t=1, \ldots, s$.

Rewrite $w=A^{m_{1}} P_{1} P_{2} \ldots P_{s-1} B^{\bar{s}}$, where $P_{t}=B^{\bar{t}} A^{m_{t+1}} B^{-\bar{t}} \in K$. Since $\bar{s}=0$, we have $w \in K$. This concludes the proof of the lemma.

Now consider $w\left(A_{i}, B_{i} ; A_{j}, B_{j}\right) \in \Gamma$ an arbitrary, nontrivial word in the generators $A_{i}, B_{i}, A_{j}, B_{j}, i=1, \ldots, p, j=p+1, \ldots, p+q$. It may be written

$$
w=w_{1}\left(A_{k_{1}}, B_{k_{1}}\right) \cdots w_{r}\left(A_{k_{r}}, B_{k_{r}}\right),
$$

where $k_{m} \in\{1, \ldots, p+q\}, m=1, \ldots, r, w_{m}$ is a nontrivial word, and $k_{m} \neq k_{m+1}$ for $m<r$.

Lemma 4. $\operatorname{ker} \chi^{*}=N$.

Proof. It is clear from Lemma 1 that $\chi_{n}\left(A_{i}\right), \chi_{n}\left(B_{i}\right)$ commute; hence, since $\chi_{n}\left(A_{j}\right)=$ id, we have that $N \subset \operatorname{ker} \chi_{n}$ for all $n \geq 0$. Thus, $N \subset \operatorname{ker} \chi^{*}$. To show the opposite inclusion, suppose $w\left(A_{i}, B_{i} ; A_{j}, B_{j}\right) \in \Gamma$ is a word with $w \notin N$. Choose $\hat{w} \in \Gamma$ to be a word which is equivalent to $w \bmod N$ and contains no subwords lying in $N$. Then, since $N \subset \operatorname{ker} \chi_{n}, \chi_{n}(w)=\chi_{n}(\hat{w})$.

We write $\hat{w}=w_{1}\left(A_{k_{1}}, B_{k_{1}}\right) \cdots w_{r}\left(A_{k_{r}}, B_{k_{r}}\right)$ as in (3.3). Then

$$
\chi_{n}(w)=\prod_{m=1}^{r} \chi_{n}\left(w_{m}\left(A_{k_{m}}, B_{k_{m}}\right)\right) .
$$

Since $\chi_{n}\left(A_{k_{m}}\right)$ commutes with $\chi_{n}\left(B_{k_{m}}\right)$, it follows from (3.4) that

$$
\chi_{n}(w)=\prod_{m=1}^{r} \chi_{n}\left(A_{k_{m}}^{m_{1}}\right) \chi_{n}\left(B_{k_{m}}^{m_{2}}\right),
$$

where $m_{1}=w_{m}^{A}, m_{2}=w_{m}^{B}$. Now $w_{m} \notin N$, hence $w_{m} \notin K_{k_{m}}$. Applying Lemmas 2 and 3, we have that for each $m$ at least one of the integers $m_{1}$ or $m_{2}$ is not 0 .

Since $\chi_{n}\left(A_{k_{m}}\right)=\chi_{n}\left(B_{k_{m}}^{-n}\right)$ for $k_{m}=1, \ldots, p$ and $\chi_{n}\left(A_{k_{m}}\right)=$ id for $k_{m}=$ $p+1, \ldots, p+q$, we obtain from (3.5) that

$$
\chi_{n}(w)=\prod_{m=1}^{r} \chi_{n}\left(B_{k_{m}}^{-n m_{1}+m_{2}}\right) .
$$

But $-n m_{1}+m_{2}=0$ for at most one value of $n$. Hence, (3.6) implies that, for almost all $n, \chi_{n}(w) \neq$ id (recall that $k_{m} \neq k_{m+1}, m<r$ ). Applying (3.1), one has that $\chi^{*}(w) \neq$ id. This concludes the proof of Lemma 4.

Proof of Theorem 1. Let $\varphi \in C_{2}(\Gamma)$ determine the normalized projective structure $\left(f_{\varphi}, \chi_{\varphi}\right)$, with $\chi_{\varphi}(\Gamma)$ an extended Schottky group. Suppose $\Gamma$ is the Fuchsian equivalent of $\chi_{\varphi}(\Gamma)$.

As we have just shown, there exists a sequence of projective structures $\left(f_{n}, \chi_{n}\right)$, with $S\left(f_{n}\right)=\varphi_{n} \in C_{2}(\Gamma)$ and $\chi_{n}(\Gamma)$ a Schottky group, converging to $\left(f, \chi^{*}\right)$ with $S(f) \in C_{2}(\Gamma)$ and $\operatorname{ker} \chi^{*}=\operatorname{ker} \chi_{\varphi}$. It is easy to show that the Kleinian group $\chi^{*}(\Gamma)$ has a connected region of discontinuity (see [7, 8] for a proof). Moreover, it is known that, for groups with a connected region of discontinuity, the conformal structure of the quotient surface plus the 
kernel of the homomorphism determine the homomorphism up to conjugation in $\operatorname{PSL}(2, \mathbf{C})$ whenever the kernel is nontrivial (see [7, 8]). Hence, since the kernels of the two homomorphisms agree and $f, f_{\varphi}$ are normalized, $\chi^{*}=\chi_{\varphi}$. It follows from the theory of projective structures that $S(f)=\varphi$ (see [5]).

Now assume that $\Gamma$ is not the Fuchsian equivalent of $\chi_{\varphi}(\Gamma)$. In this situation, there exist a finitely generated Fuchsian group $F$ with $\Gamma \subset F$ and a projective structure $\left(f_{\varphi}, \hat{\chi}\right)$ on $U / F$, where $\hat{\chi} \mid \Gamma=\chi_{\varphi}, \hat{\chi}(F)=\chi_{\varphi}(\Gamma)$, and $F$ is the Fuchsian equivalent of $\hat{\chi}(F)$ (see [7]). As before, choose $\varphi_{n} \in C_{2}(F)$ with $f_{\varphi_{n}} \rightarrow f_{\varphi}, \chi_{\varphi_{n}} \rightarrow \hat{\chi}$. Let $\chi_{\varphi_{n}}^{\prime}=\chi_{\varphi_{n}} \mid \Gamma$. Now $\left(f_{\varphi_{n}}, \chi_{\varphi_{n}}^{\prime}\right)$ is a projective structure on $\Gamma$, and $\chi_{\varphi_{n}}^{\prime}(\Gamma)$, being a finitely generated subgroup of a Schottky group, is a Schottky group. Since $\chi_{\varphi_{n}}^{\prime} \rightarrow \hat{\chi} \mid \Gamma=\chi_{\varphi}$, the differentials $\varphi_{n}$ (note that $\varphi_{n} \in C_{2}(\Gamma)$, also) have the required properties.

\section{SChotTKY STRUCTURES IN GENUS 1}

We consider now a compact Riemann surface $T$ of genus 1 . It is conformally equivalent to $\mathrm{C} / G$, where $G$ is a rank two parabolic group with generators $A(z)=z+1, B(z)=z+\omega$, and $\omega \in \mathbf{C}$ has positive imaginary part.

In this situation, a projective structure $(f, \chi)$ consists of a holomorphic local homeomorphism $f: \mathbf{C} \rightarrow \mathbf{C}$ and a homomorphism $\chi: G \rightarrow A(2, \mathbf{C})$, where $f \circ \gamma=\chi(\gamma) \circ f$. Here, $A(2, \mathbf{C})$ is the affine group consisting of all elements of $\operatorname{PSL}(2, \mathrm{C})$ fixing $\infty$.

Following Gunning [1], $f^{\prime \prime} / f^{\prime}=2 \pi i c$ for some constant $c \in \mathbf{C}$, and the projective structures on $T$ can be parametrized by $C$. For $c=0$, we will normalize so that $f_{0}(z)=z$ and $\chi_{0}$ is the identity homomorphism. If $c \neq 0$, $f(z)=a e^{2 \pi i c z}+b$ where $a \neq 0$, and we precompose $f$ by an affine transformation so that $f_{c}(z)=e^{2 \pi i c z}$ and $\chi_{c}(A)(z)=e^{2 \pi i c} z, \chi_{c}(B)(z)=e^{2 \pi i c \omega} z$. (Note that all structures are given by covering maps.)

One sees easily that, for $n>0, c_{n}=1 /(\omega-n)$ satisfies $\left|e^{2 \pi i c_{n}}\right| \neq 1$, with

$$
\chi_{c_{n}}(A)(z)=e^{2 \pi i /(\omega-n)} z, \quad \chi_{c_{n}}(B)(z)=e^{2 \pi i n /(\omega-n)} z .
$$

Thus, $\chi_{c_{n}}(G)=\left\langle\chi_{c_{n}}(A), \chi_{c_{n}}(B)\right\rangle$ is a purely loxodromic, discontinuous group generated by $\chi_{c_{n}}(A)$. Moreover, $(\mathbf{C}-\{0\}) / \chi_{c_{n}}(G)=T$. Extending the definition of $\S 2$, we will say that $\chi_{c_{n}}(G)$ is a Schottky group. Note that $c_{n} \rightarrow 0$, but $\chi_{c_{n}}$ does not converge algebraically to $\chi_{0}$.

In order to obtain algebraic convergence, we renormalize in the following manner: Define $\beta_{n} \in A(2, \mathbf{C})$ as $\beta_{n}(z)=z+b_{n}$, where $b_{n}=n e^{-2 \pi i /(w-n)} / 2 \pi i$. Now, consider the structures $\left(g_{n}, \chi_{n}\right)$, where $g_{n}=\beta_{n} \circ f_{c_{n}}, \chi_{n}=\beta_{n} \circ \chi_{c_{n}} \circ \beta_{n}^{-1}$. Then

$$
\chi_{n}(A)(z)=e^{2 \pi i /(\omega-n)} z-b_{n}\left(e^{2 \pi i /(\omega-n)}-1\right)
$$

and

$$
\chi_{n}(B)(z)=e^{2 \pi i n /(\omega-n)} z-b_{n}\left(e^{2 \pi i n /(\omega-n)}-1\right) .
$$

It is straightforward to verify that $\chi_{n}(A)(z) \rightarrow z+1$ and that $\chi_{n}(B)(z) \rightarrow$ $z+\omega$. Observe that $g_{n}(z)=e^{2 \pi i z /(\omega-n)}+n e^{-2 \pi i /(\omega-n)} / 2 \pi i$ diverges.

In general, for an arbitrary sequence $\left(h_{c_{k}}, \chi_{c_{k}}\right)$ with $c_{k} \rightarrow 0$, one cannot expect $h_{c_{k}}$ to converge to a covering map. Note that, necessarily, $h_{c_{k}}(z)=$ $a_{k} e^{2 \pi i c_{k} z}+b_{k}$ and $e^{2 \pi i c_{k} z}$ converges to a constant map.

Nonetheless, we have proven the following analogue to Theorem 1: 
Theorem 2. Let $G$ be a rank 2 parabolic group acting on $\mathbf{C}$. Let $\left(f_{0}, \chi_{0}\right)$ be the identity structure on $\mathbf{C} / G$; that is, $f_{0}(z)=z$ and $\chi_{0}$ is the identity homomorphism. Then there exists a sequence of projective structures $\left(f_{n}, \chi_{n}\right)$ on $\mathbf{C} / G$, where $\chi_{n}(G)$ is a Schottky group and $\chi_{n}$ converges algebraically to $\chi_{0}$.

\section{REFERENCES}

1. R. C. Gunning, Affine and projective structures on Riemann surfaces, Proc. 1978 Stony Brook Conference, Ann. of Math. Stud., no. 97, Princeton Univ. Press, Princeton, NJ, 1981, pp. 225-244.

2. D. Hejhal, On Schottky and Teichmuller spaces, Adv. Math. 15 (1975), 133-156.

3. T. Jorgensen, On discrete groups of Möbius transformations, Amer. J. Math. 98 (1976), 739-749.

4. T. Jorgensen and P. Klein, Algebraic convergence of finitely generated Kleinian groups, Quart. J. Math. Oxford Ser. (2) 33 (1982), 325-332.

5. I. Kra, Deformations of Fuchsian groups. II, Duke Math. J. 38 (1971), 499-508.

6. __ Families of univalent functions and Kleinian groups, Israel J. Math. 60 (1987), 89-127.

7. I. Kra and B. Maskit, Remarks on projective structures, Proc. 1978 Stony Brook Conference, Ann. of Math. Stud., no. 97, Princeton Univ. Press, Princeton, NJ, 1981, pp. 343-359.

8. B. Maskit, Decomposition of certain Kleinian groups, Acta Math. 130 (1973), 243-263.

9. W. P. Thurston, Geometry and topology of 3-manifolds, Princeton lecture notes, 1979.

Department of Mathematics, St. John's University, Staten Island, New York 10301 E-mail address: gallo@sjuvm.bitnet 